Cognitive Behavior Therapy for Comorbid Migraine and/or Tension-type Headache and Major Depressive Disorder: An Exploratory Randomized Controlled Trial

\author{
Paul R Martin ${ }^{\mathrm{a}^{*}}$, Rachele Aiello ${ }^{\mathrm{b}}$, Kathryn Gilson ${ }^{\mathrm{b}}$, \\ Graham Meadows $^{\mathrm{b}}$, Jeannette Milgrom ${ }^{\mathrm{c}}$ and John Reece ${ }^{\mathrm{d}}$
}

a School of Applied Psychology and Menzies Health Institute Queensland, Griffith University, Mt Gravatt Campus, 176 Messines Ridge Road, Mt Gravatt, Queensland 4122, Australia.

b School of Psychology and Psychiatry, Monash University, Monash Medical Centre, 246 Clayton Road, Victoria 3168, Australia.

${ }^{\mathrm{c}}$ Melbourne School of Psychological Sciences, University of Melbourne, and Heidelberg Repatriation Hospital, 300 Waterdale Road, Heidelberg Heights, Victoria, 3081, Australia. ${ }^{\mathrm{d}}$ School of Health Sciences, RMIT University, PO Box 71, Bundoora, Victoria 3083, and Australian College of Applied Psychology, Australia.

*Corresponding author. Tel: +61 (0)7 3735 3322; fax: +61 (0)7 3735 3399; email: paul.martin@griffith.edu.au.

\begin{abstract}
Abbreviations: TTH, tension-type headache; EMG, Electromyographic; CBT, cognitive behavior therapy; RCT, randomized controlled trial; CONSORT, Consolidated Standards of Reporting Trials; RPC, routine primary care; CIDI-LT, Composite International Diagnostic Interview 2.1 Auto Lifetime version; BDI-II, Beck Depression Inventory; BAI, Beck Anxiety Inventory; PHQ-9, Patient Health Questionnaire; AQoL, Australian Quality of Life instrument; FQ, Feedback Questionnaire.
\end{abstract}




\begin{abstract}
Numerous studies have demonstrated comorbidity between migraine and tension-type headache on the one hand, and depression on the other. Presence of depression is a negative prognostic indicator for behavioral treatment of headaches. Despite the recognised comorbidity, there is a limited research literature evaluating interventions designed for comorbid headaches and depression. Sixty six participants (49 female, 17 male) suffering from migraine and/or tension-type headache and major depressive disorder were randomly allocated to a Routine Primary Care control group or a Cognitive Behavior Therapy group that also received routine primary care. The treatment program involved 12 weekly 50minute sessions administered by clinical psychologists. Participants in the treatment group improved significantly more than participants in the control group from pre- to post-treatment on measures of headaches, depression, anxiety, and quality of life. Improvements achieved with treatment were maintained at four month follow-up. Comorbid anxiety disorders were not a predictor of response to treatment, and the only significant predictor was gender (men improved more than women). The new integrated treatment program appears promising and worthy of further investigation.
\end{abstract}

Keywords: migraine, tension-type headache, depression, cognitive behavior therapy. 


\section{Cognitive Behavior Therapy for Comorbid Migraine and/or Tension-type Headache and Major Depressive Disorder: An Exploratory Randomized Controlled Trial}

Headache and psychiatric comorbidity has received much attention, including a Supplement of Headache devoted to it (Headache 2006, 46[Suppl 3]). The main focus of this literature has been on migraine, and investigations have confirmed the association between migraine and a number of psychiatric disorders, in particularly major depressive disorder and anxiety disorders (Radat \& Swendsen, 2004; Baskin \& Smitherman, 2011; Hamelsky \& Lipton, 2006; Guidetti et al., 1998). Estimates of the increased risk of depression for individuals suffering from migraine range from 2.2 to 4.0 times more likely (Hamelsky \& Lipton, 2006; Lake, Rains, Penzien, \& Lipchik, 2005; Lipchik \& Penzien, 2004; Breslau, Lipton, Stewart, Schultz, \& Welch, 2003). Anxiety disorders, such as generalized anxiety disorder, panic disorder, phobia and obsessive-compulsive disorder, occur three to five times more frequently in the migraine population than in the general population (Radat \& Swendsen, 2004; Hamelsky \& Lipton, 2006). Comorbid migraine and depression has a number of negative consequences including decreased quality of life (Pompili et al., 2009), and increased risk of suicide, medication overuse, and disability (Buse \& Andrasik, 2009; Smitherman, McDermott, \& Buchanan, 2011; Lake, 2006; Bigal \& Lipton, 2006).

The relationship between psychiatric comorbidity and headache is not limited to migraine. Chronic daily headache has been associated with higher levels of depressive and anxiety disorders than has episodic migraine (Radat \& Swendsen, 2004; Lipchik, Smitherman, Penzien, \& Holroyd, 2006) (chronic daily headache includes chronic migraine and chronic tension-type headache). A study has reported elevated major depressive disorder and anxiety disorders in chronic tension-type headache (Juang, Wang, Fuh, Lu, \& Su, 2000), and studies comparing frequency of psychiatric disorders between migraine and tension-type headache (TTH) groups have failed to find differences (Marazziti et al., 1995). 
Comorbidity between headaches and depression could reflect a causal relationship whereby headaches cause depression, or depression causes headaches. Alternatively, the relationship may not be causal with other factors explaining the association, such as trauma/stress leading to both disorders, or common genetic predispositional factors resulting in high co-occurrence. For example, one study estimated that $20 \%$ of the variance in migraine and depression could be explained by shared genetics (Schur, Noonan, Buchwald, Goldberg, \& Afari, 2009). These explanations are not mutually exclusive, and it seems plausible that a combination of factors is likely to explain comorbidity. Cross-sectional retrospective studies have demonstrated a tendency for depression to follow headaches (Breslau, Davis, \& Andreski, 1991; Radat et al., 2005). A major longitudinal study demonstrated that migraine predicted depression at twice the rate that depression predicted migraine (Breslau et al., 2003). A second longitudinal study demonstrated that depression followed migraine in $55 \%$ of cases (Breslau, Davis, Schultz, \& Paterson, 1994). Wynd, Martin, Gilson and Meadows (in press) found that $87 \%$ of a sample of individuals with comorbid headache and major depressive disorder reported that life events such as being bullied at work, death of a family member, and moving to another country, contributed to both disorders.

Many studies have shown that psychological treatment for headache is efficacious. For example, Rains, Penzien, McCrory and Gray (2005) summarized the results of five metaanalytic reviews for psychological treatment (thermal biofeedback, electromyographic [EMG] biofeedback, cognitive behavior therapy [CBT], relaxation training) of migraine published between 1980 and 1999, and concluded that average improvement ranged from $33 \%$ to $55 \%$, compared with 5\% for no-treatment controls. These authors also summarized the results of four meta-analytic reviews for psychological treatment (EMG biofeedback, CBT, relaxation training) of TTH, and concluded that average improvements ranged between $35 \%$ and 55\%, compared with $2 \%$ for no-treatment controls. 
Headache sufferers who also experience psychiatric disorders respond less well to treatment. A longitudinal study suggested that the presence of psychiatric disorders may predict poorer outcome for headache treatment especially if multiple psychiatric disorders are present (Guidetti et al., 1998). This study found that 86\% of those with headache who had two or more comorbid psychiatric disorders in childhood or adolescence had either no improvement or deterioration in their headache condition (migraine or TTH). In 62\% of patients with a single psychiatric disorder in childhood or adolescence, migraine remained unchanged or worsened. In contrast, the absence of psychiatric disorders was associated with remission of headaches after 8 years. Another study of migraine treatment suggested that presence of comorbid psychiatric disorders may be associated with long-term (6 to 7 years) relapse, even if it is not associated with a poor initial response (Mongini et al., 2003).

The efficacy of psychological treatments for depression is well established. Butler, Chapman, Forman and Beck (2006) reviewed meta-analyses of CBT and found large effect sizes for CBT for unipolar depression. They also reported that CBT was superior to antidepressants in the treatment of adult depression. Others have suggested that CBT is similar in effectiveness to tricyclic antidepressants (American Psychiatric Association, 2000). There is evidence that the effects of CBT on depression may be more enduring than antidepressant medications (Hollon \& Ponniah, 2010; Driesen \& Hollon, 2010).

As it is well established that headaches and depression are comorbid, that depression is a negative prognostic indicator, and that guidelines have been offered as to how to treat these patients from a CBT perspective (Lake, 2006; Lipchik et al., 2006), it is surprising that limited research has been reported investigating the effectiveness of such approaches. A recent study did evaluate a one-day behavioral intervention based on Acceptance and Commitment Training plus Migraine Education for depressed migraine patients (Dindo, Recober, Marchman, O’Hara, \& Turvey, 2014; Dindo, Recober, Marchman, Turvey, \& 
O’Hara, 2012). The study compared the intervention with 'wait list/treatment as usual' and reported that participants who received the intervention exhibited significant improvements in headache frequency, headache severity, medication use and headache-related disability at three month follow-up. The control group did not show improvement over time, but the difference in outcomes between the two groups was not statistically significant (Dindo et al., 2014). Participants in the intervention group exhibited significantly greater improvements in depressive symptoms than participants in the control group (Dindo et al., 2012).

The goal of the study reported here was to develop an integrated CBT approach for individuals suffering from recurrent headaches and major depressive disorder. The headache component of the program drew on the book on psychological treatment of headaches by one of the authors (Martin, 1993) which describes a version of CBT based on a functional model of primary headaches. This model seeks to understand the variance in headache activity by considering the antecedents and consequences of headaches, and has been described in a number of publications, most recently Martin (2013). This approach has been shown to be effective as it was associated with the following changes in an RCT: (i) average decrease in headaches of $68 \%$ post-treatment, and $77 \%$ at 12 month follow-up; and (ii) average decrease in medication of 70\% post-treatment (Martin, Forsyth, \& Reece, 2007). The depression component of the program drew on Peter Lewinsohn's “Coping with Depression Course” (Lewinsohn, Antonuccio, Steinmetz, \& Teri, 1984). This CBT approach to depression was selected on the basis that the authors have extensive experience with it including adapting it for the treatment of postnatal depression (Milgrom, Martin, \& Negri, 1999).

The main aim of the study was to evaluate the new integrated approach to treating comorbid headaches and depression. A secondary aim was to examine predictors of response to treatment. For example, would presence of one or more comorbid anxiety disorders adversely affect response to treatment? 


\section{Methods}

\section{Design}

The study employed a split-plot ANOVA design, consisting of one between-subjects factor (Group), and one within-subjects factor (Time). A randomization procedure based on the CONSORT guidelines was used to allocate participants to a treatment group (CBT for recurrent headache and comorbid depression) or control group (routine primary care, RPC). General considerations in the study design were to maximize practicability of the intervention studied for uptake in routine practice by typical practitioners, so the study can be seen as a phase 1-2 translational research design (Lean, Mann, Hoek, Elliot, \& Schofield, 2008).

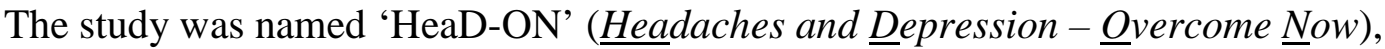
and was approved by the Southern Health Human Research Ethics Committee (Number: 08062A). Medicare Australia (the government healthcare system) was approached about use of Medicare rebates in the study and the response indicated that this would be acceptable for the clinical care component of the research. This enabled the treatment to be delivered in line with available rebates, which in turn provided good alignment with phase 2 translational research considerations of practicability of implementation in routine clinical work. This trial was registered with the Australian New Zealand Clinical Trials Registry (ANZCTR: 12608000164392).

\section{Participant Selection Criteria, Recruitment and Flow}

Inclusion criteria: (i) diagnosed as suffering from either 'typical aura with migraine headache', 'migraine without aura', 'chronic migraine’, 'frequent episodic tension-type headache', or 'chronic tension-type headache' (Headache Classification Subcommittee of IHS, 2004); (ii) minimum of 6 headache days per month; (iii) minimum of 12 months with this frequency of headaches, and pattern of headache symptoms stable over last 6 months; (iv) diagnosed as suffering from 'major depressive disorder' (World Health Organization 
Collaborating Centre for Mental Health and Substance Abuse, 1997); and (v) aged 18 years or over.

Exclusion criteria. In accordance with the Guidelines for Trials of Behavioral Treatments for Recurrent Headache (Penzien et al., 2005), the intention was to exclude individuals with substantial medical or psychiatric comorbidities, except major depressive disorder and anxiety disorders. Time constraints, however, limited completion of a full diagnostic assessment to depressive and anxiety disorders. Other obvious exclusion criteria were used, such as factors interfering with giving informed consent or benefiting from treatment (e.g., poor English, intellectual disability).

Participants were recruited from four Divisions of General Practice in the Southern Health catchment area. In addition, posters were used and media releases resulting in recruiting articles in newspapers, and radio interviews. Participant flow through the study is summarized in the CONSORT Flow Diagram in Figure 1. The top box in the diagram is 'first contact', and reflecting our recruitment procedures, could come to us at the University directly from a potential participant or from a referring doctor, via letter, email or phone.

\section{Measures}

Headaches and medication consumption. Participants completed diaries by recording hourly ratings of headache intensity throughout the waking day using a scale from 0 (no headache) to 5 (an intense incapacitating headache). This 'time-sampling' procedure has well-established reliability (Collins \& Thompson, 1979) and validity (Blanchard, Andrasik, Neff, Jurish, \& O’Keefe, 1981) and is generally regarded as the 'gold standard' in behavioral headache research (Andrasik, Lipchik, McCrory, \& Whitrock, 2005). Medication consumption was also recorded in daily diaries (type, dosage and time taken). A system was developed using KeyPoint version 5.5 (Cambridge Software Publishing) to electronically scan the daily diaries and export the data to an SPSS file. 
Mood and anxiety disorders were diagnosed using the Composite International Diagnostic Interview 2.1 Auto Lifetime version (CIDI-LT) (World Health Organization Collaborating Centre for Mental Health and Substance Abuse, 1997). The CIDI was developed as a collaborative project between the World Health Organization and the US National Institutes of Health. It is fully standardized and assesses mental disorders according to the definitions and criteria of the International Classification of Diseases-10 and the Diagnostic and Statistical Manual of Mental Disorders-IV. Only the modules for depressive disorders and anxiety disorders were used in the study.

Depression and anxiety. Severity of depression was measured with the revised Beck Depression Inventory (BDI-II) (Beck \& Steer, 1987). Anxiety was measured with the Beck Anxiety Inventory (BAI) (Beck, Epstein, Brown, \& Steer, 1988), designed for use in conjunction with the BDI. The BDI-II and BAI are used very extensively in research studies and have well established psychometric properties. Depression was also measured using the Patient Health Questionnaire (PHQ-9), a brief screening questionnaire that is a reliable and valid measure of depression severity (Kroenke, Spitzer, \& Williams, 2001).

Quality of life was measured using the complementary SF-36 Health Survey from the Medical Outcomes Study (Ware, Gandek, IQOLA Project Group, 1994) and Australian Quality of Life instrument (AQoL) (Hawthorne, Richardson, \& Osborne, 1999). The SF-36 has been proven valid through numerous studies. The AQoF has high internal consistency (alpha $=0.81)$ and good construct validity.

Feedback Questionnaire (FQ). Participant perceptions of the program were assessed by 20 questions that asked how useful they found different components of the program, and an open-ended question about how they benefited from the program.

\section{Treatment Groups}

CBT. The treatment program developed involved 12, 50-minute individual sessions 
scheduled weekly. The CBT program for headaches that served as the starting point for the integrated program involved eight sessions (Martin et al., 2007), but it was possible to develop a program targeting both headaches and depression with only a 50\% increase in sessions because of the overlap between the two treatment programs that were combined. The program was described in a 144 page Treatment Manual that included therapist notes, client handouts and a relaxation CD (Nejad \& Volny, 2008). Participants in this condition also received a 108 page Client Handbook.

The treatment program contained the following elements: (i) education about headaches and depression; (ii) relaxation training (progressive muscle relaxation); (iii) scheduling pleasant activities; (iv) social skills training (assertiveness and self-esteem); (v) training to challenge dysfunctional thoughts and beliefs; (vi) problem solving training; (vii) identifying and managing headache triggers; (viii) training in pain management techniques (imagery training and attention-diversion training); (ix) lifestyle management (exercise, social support, sleep); and (x) relapse prevention training. The Treatment Manual and Client Handbook will be provided on request by the authors, firstly so that the intervention can be considered in detail in understanding findings of this study, secondly so that it is readily available for clinical use if required, and finally to assist others who may wish to mount further phase 2 and 3 (Lean et al., 2008) translational research.

For participants in this group, no attempt was made to restrict GPs in terms of prescribed medication (all medications consumed by participants were recorded by them in the diaries). GPs were requested to restrict the use of CBT to the CBT administered through the study, and to not refer to psychologists not involved in the study (no attempt was made to restrict other referrals, for example, to neurologists or psychiatrists).

$\boldsymbol{R P C}$. Participants in this group were case-managed by their GPs as they would in the absence of the study, with small variations. No attempt was made to restrict GPs in terms of 
prescribed medication. GPs were requested to not use CBT, and to not refer to psychologists not involved in the study (no attempt to restrict other referrals was made, for example, to neurologists or psychiatrists). Other variations from routine care include the training of the GPs in detecting comorbidity, and the assessments and self-monitoring carried out by the participants. After these participants had completed their 'post-treatment' assessment, they were offered the same treatment as participants in the other group.

\section{Clinical Psychologists Conducting the Study}

Recruitment, assessment and training in self-monitoring were carried out by staff employed on the grant at the University (Monash Medical Centre). These staff were doctoraltrained clinical psychologists. In preparation for the study they attended a one-day workshop on headaches presented by the primary author who has 40 years of experience as a researcher, clinician and educator in the headache domain. The staff met weekly with the primary author for supervision throughout the study.

Treatment was carried out by clinical psychologists working in independent practice in the geographical area of the trial. All of the therapists had completed postgraduate training in clinical psychology and a minimum of two years supervised practice after completing their training. All of the therapists completed the same one-day workshop on headaches as the clinical psychologists carrying out the assessments. This workshop has been presented at international conferences in North America (USA and Canada), Europe, Asia and Australia. It is primarily didactic, with extensive opportunity provided for discussion. It is based on the principle that the techniques used with headache sufferers are not unique to this problem and hence the need is not for learning new techniques. What is required is learning about how to apply these techniques to headaches, and most importantly acquiring a model (the functional model of headaches) that helps headache sufferers understand their headaches, and provides a platform for the assessment and treatment of headaches. The workshop was endorsed by the 
Australian Psychological Society for professional development credit for registrants.

To be involved in the study, the psychologists had to agree to follow all the study protocols including the Treatment Manual. They could all contact the research team for help and support at any time. They were also required to complete checklists of what they completed in the treatment sessions to encourage adherence. Twenty nine ( 24 female, 5 male) clinical psychologists provided treatment in the study. The mean number of years since completing training was 10.9 years $(S D=8.79)$, and $37.5 \%$ had treated a client for headaches before becoming involved in the research. Treatment was administered in the practices of the psychologists, and Medicare Australia was bulk billed for the treatment sessions.

\section{Procedure}

Pre-treatment assessment and training. Individuals referred to the study were invited to a pre-treatment assessment session at which informed consent procedures were completed. An assessment followed that collected information pertaining to selection criteria. Headache diagnosis was completed using a structured interview based on the International Classification of Headache disorders, $2^{\text {nd }}$ edition (Headache Classification Subcommittee of IHS, 2004). The CIDI-LT was used for diagnosing major depressive disorder and anxiety disorders (World Health Organization Collaborating Centre for Mental Health and Substance Abuse, 1997). Participation ceased at this point for all individuals who did not meet the criteria and they were provided with information pertaining to referral sources. For eligible individuals, demographic information was recorded. Participants then completed the BDI-II, BAI, PHQ-9, SF-36 and AQoL. The latter part of the session was used for training in selfmonitoring headaches and medication consumption.

Baseline self-monitoring. Participants commenced self-monitoring the day after the pre-treatment assessment session and continued for two weeks before treatment began. Participants completed the BDI-II and BAI again at the end of this period. 
Randomization. During the two week period of self-monitoring, participants were randomly allocated to one of the two groups in the study. One of the authors with no involvement with participants took responsibility for the randomization. A computergenerated sequence was used, and the CONSORT guidelines (http:www.consortstatement.org/Statement/revisedstatement.htm) followed in full. Participants assigned to the CBT group were then allocated to the clinical psychologist in the study whose practice was most convenient for attendance.

Treatment. Participants in the CBT group received treatment over the next 12 weeks whilst no additional treatment was provided for participants in RPC. All participants continued self-monitoring headaches and medication throughout this period, and completed the BDI II and BAI every four weeks.

Post-treatment assessment. All participants continued self-monitoring headaches and medication for two weeks after the treatment period. Participants then completed the BDI-II, BAI, PHQ-9, SF-36, AQoL and FQ. At the completion of this period, participants in the RPC group were offered the CBT program.

Four month follow-up assessment. Four months after completing treatment, participants were asked to resume self-monitoring headaches and medication use for a two week period. They were requested to complete the BDI-II, BAI, PHQ-9, SF-36 and AQoL.

\section{Financial Implications of Taking Part in the Study for Participants}

Participants received vouchers redeemable at a local supermarket chain for reimbursement of expenses (time and transport/parking costs) involved in attending assessment sessions. The vouchers were for A \$20 except for attendance at the pre-treatment assessment session which was a much longer session, for which the vouchers were for $\mathrm{A} \$ 50$.

No reimbursements were made for attending treatment sessions. Hence reimbursements were provided for sessions that had research objectives but not sessions that 
had clinical objectives. Because the treating psychologists bulk billed Medicare, there were no costs for treatment to the participants.

Analysis strategy. Analysis of the main outcome variables was based around splitplot factorial analysis of variance using linear mixed models. All analyses were univariate (linear mixed models does not accommodate multivariate analysis). For each analysis, the between-subjects factor was treatment (CBT versus RPC) and the within-subjects factor was phase, which varied across measures, depending on how many phases of data were analyzed. For example, for mean daily headache ratings, four phases of data collection took place for the CBT group, but only three for RPC, whereas for other outcome measures, data for both groups were analyzed for the baseline and post-treatment phases.

Linear mixed modelling was chosen over multivariate or general linear modelling for several reasons: (i) linear mixed modelling easily accommodates missing cells, of which there were many in these data; (ii) linear mixed modelling deals efficiently with missing data from within-subjects factors without the need for data imputation; and (iii) linear mixed modelling allows a range of covariance structures from within-subjects effects to be modelled. In the case of the analyses reported here, the commonly used Akaike information and Bayesian information criteria indicated that a compound symmetry covariance structure provided the best model fit for all outcome variables.

For each outcome variable, the treatment by phase interaction was the central test of importance. Significant interactions were followed by simple main effects tests, with a focus on the treatment within phase effect, which are presented in Table 3. For all inferential tests, an effect size is reported (either Cohen's $d$ or $\eta_{\mathrm{p}}{ }^{2}$ ) with an associated 95\% confidence interval. Sidak correction for multiple testing was carried out automatically by SPSS.

It was initially intended to analyse the data on an intention-to-treat basis; however, the amount of missing data and its non-random nature rendered that impossible. Instead the 
data were analyzed on an available case basis (Altman, 2009) using linear mixed models, which allowed all available data to be included in the analyses. This accounts for the figures in the analysis section of the CONSORT flow chart in Figure 1. Despite the loss of participants at post-assessment and follow-up, all randomized participants were included in the analysis.

Multiple imputation for missing data was considered, but given the amount of missing data, particularly for the follow-up phases, and its non-random nature, it was felt that any form of missing value estimation was unwarranted, including last observation carried forward. This was supported by visual inspection of the pattern matrices for missing data, along with the results of Missing Completely at Random tests. Further support for this approach was obtained by an analysis of drop-outs, which involved comparing participants who completed the treatment protocol with those who provided baseline data but did not complete. Using independent samples $t$-tests and $\chi^{2}$ analysis, no significant differences were found between completers and non-completers on all major variables.

\section{Results}

Participant characteristics. Demographic and clinical characteristics of the sample are shown in Table 1. Occupations have been classified according to the Australian and New Zealand Standard Classification of Occupations. One type of headache was identified for 20 participants and two types for 46 participants. The headache diagnoses in Table 1 are the primary diagnoses. Anxiety disorders were diagnosed in 47 participants, and a total of 96 anxiety disorders were identified. Posttraumatic Stress Disorder and Specific Phobia were the most common anxiety diagnoses, but Panic Disorder With and Without Agoraphobia, and Social Phobia, were also common. Most frequently, participants were diagnosed with one $(n=18)$ or two anxiety disorders $(n=15)$, but two participants were diagnosed with four anxiety disorders each, and two participants with five anxiety disorders each. 
Independent samples $t$-tests found no significant difference between the CBT and RPC groups on age, headache chronicity, or baseline depression. Further, $\chi^{2}$ analysis found no significant association between treatment group and primary headache diagnosis (migraine versus TTH), gender, or whether a comorbid anxiety disorder was present.

Data preparation and assumption testing. Data were collected between July 2008 and January 2012. Headache intensity ratings from the diaries were averaged across the waking day to produce a composite index (mean daily headache ratings) that reflected the three headache parameters of intensity, duration and frequency. Medication scores (pill counts) from the diaries were added for each day (mean daily medication use) as recommended by the International Headache Society (International Headache Society Committee on Clinical Trials in Tension-type Headache, 2000). Individual values for these scores along with scores for all of the other measures described above, were entered for each participant at each of the four experimental phases into a single SPSS 21 spreadsheet.

All outcome variables were assessed for normality and homogeneity of variance, along with a range of other assumptions related to the use of linear mixed models (e.g., linearity, homoscedasticity, etc.). All variables exhibited some degree of non-normality as assessed by visual inspection of histograms, stem-and-leaf and normality plots, and normality tests, but the degree of non-normality was not considered serious enough to warrant transformation or the use of non-parametric procedures, particularly given the sample size and the robustness of the chosen procedures to deviations from normality (Norman, 2010).

Heterogeneity of variance was evident for several variables. In each instance, a power transformation based on the power figure recommended by SPSS 21 resulted in the assumption being met. For the analyses presented below, descriptive statistics are reported using the original metric and all inferential tests are based on the transformed data. Other assumption tests all revealed results within tolerable bounds. 
Primary aim - effectiveness of treatment. Table 2 presents the results for all outcome variables. Focusing on the primary outcome measures, that is, the measures of headaches and depression, it can be seen that mean daily headache ratings decreased from baseline to posttreatment by $41.7 \%$ in the CBT group compared with $1.9 \%$ in the RPC group, and this interaction was significant at $p=.02$. These results are presented graphically in Figure 2 . The two measures of depression severity were the BDI-II and PHQ-9. BDI-II scores decreased from baseline to post-treatment by $57.1 \%$ in the CBT group compared to $13.4 \%$ in the RPC group, and this interaction was highly significant at $p<.001$. PHQ-9 scores decreased from baseline to post-treatment by $61.5 \%$ in the CBT group compared to $27.8 \%$ in the RPC group, and this interaction was also highly significant at $p<.001$. The depression results are presented graphically in Figure 3.

The pattern of findings in Figures 2 and 3 is representative of the findings for all the other significant secondary outcome measures. Notably, on the measure of anxiety, BAI scores decreased from baseline to post-treatment by $49.4 \%$ in the CBT group compared with $13.9 \%$ in the RPC group, and this interaction was significant at $p<.001$. There were also significant interactions on measures of quality of life, specifically for the Australian Quality of Life instrument (AQoL), and five of the eight sub-scales of the SF-36. Mean daily medication use decreased from baseline to post-treatment more in the CBT group (23.9\%) than the RPC group (16.3\%), but this interaction was not significant.

The results for the treatment within phase simple main effects analysis are provided in Table 3. For each variable the $p$ value associated with the test is provided, along with the effect size, $d$, and a 95\% confidence interval around $d$. The $p$ values for these tests are Sidak adjusted. Most notably, there were no significant differences between the RPC and CBT groups at baseline. In contrast, at post-treatment, 9 out of 10 tests were significant. 
The result for the AQoL is worthy of comment. Despite the lack of a significant simple main effect result at either baseline or post-treatment, examination of the means associated with this variable indicates that there was a notable difference between the groups at baseline, with the RPC condition demonstrating a higher quality of life, which reversed at post-treatment. This explains the significant interaction, despite the absence of a significant treatment within phase simple main effect. The phase within treatment simple main effect, although not reported here, was highly significant for the CBT group for this variable.

With respect to the treatment responder rate (proportion of participants deemed 'clinically improved'), five out of 25 (20\%) participants in the RPC group demonstrated at least a 50\% reduction in mean daily headache ratings versus 8 out of 18 (44\%) participants in the CBT group. Despite this notable difference in the percentage of participants reaching the clinical significance criterion in the two groups, a 2 × $2 \chi^{2}$ contingency table analysis did not reveal a significant association between treatment and the number of participants in each group, $\chi^{2}(1, N=43)=2.97, p=.09$.

A significant result was found, however, on an identical analysis of depression scores based on the BDI-II. For this outcome, 11 of 18 (61\%) of participants in the CBT group demonstrated at least a 50\% reduction in depression scores from baseline to post-treatment, versus two out of 26 (7\%) participants in the RPC group, $\chi^{2}(1, N=44)=14.58, p<.001$. Observation of standardized residuals revealed that the low number of participants improving in the RPC group (-2.1) and the high number of participants improving in the CBT group (2.5) contributed meaningfully to the significant outcome.

Although the phase within treatment simple main effects are not reported in detail here, there is one comparison within that analysis worthy of mention. Maintenance of treatment efficacy from post-treatment to four month follow-up was assessed by examining the post-treatment to four month follow-up pairwise comparison from the phase within 
treatment simple main effect test for the treatment group (follow-up data were collected from the treatment group only). Across all outcome variables, both primary and secondary, only one significant change from post-treatment to follow-up was observed: scores on the Vitality sub-scale of the SF-36 showed a significant continued improvement from post-treatment to follow-up, $p=.006$. At the descriptive level, it is worth noting that the majority of the primary and secondary outcome variables revealed continued improvement from posttreatment to follow-up for the treatment group. There was a slight increase in mean daily headache ratings, a small decrease in scores on the SF-36 Physical Functioning sub-scale, and trivial changes to the AQoL and the SF-36 General Health sub-scale.

Secondary aim - predictors of response to treatment. The correlations between changes in headaches and changes in the two measures of depression were investigated, with no significant outcomes. The correlations were, $r=.34, p=.22$, for BDI-II and, $r=-.16, p=$ .57, for PHQ-9. Correlations were calculated between changes in headache and changes in depression between baseline and post-treatment, and the following potential predictor variables: Baseline depression (BDI-II and PHQ-9), age, and headache chronicity. None of the potential predictor variables predicted changes in headaches or depression. The only correlations that achieved statistical significance were the obvious and trivial ones; that is, the two depression measures at baseline, $r=.54, p=.001$, the two change in depression measures, $r=.70, p=.001$, and age and headache chronicity, $r=.43, p=.009$.

Additional potential predictor variables were analyzed. Specifically, headache diagnosis (migraine versus TTH), gender, and presence or absence of one or more comorbid anxiety disorders, were investigated as predictors of changes in headaches and depression. Despite many of the descriptive results trending in a theoretically expected direction with associated moderate effect sizes, independent samples $t$-tests revealed only one significant 
finding: men demonstrated a significantly larger reduction in mean daily headaches than women, $t(16)=2.30, p=.004, d=1.21,95 \%$ CI $d[0.08,2.31]$.

Participant feedback. The mean response to the question on overall usefulness of the program was 8.25 ( $S D=2.62)$, on a scale from 0 ('not useful'), through 5 ('moderately useful'), to 10 ('very useful'). All components of the program achieved a mean score in excess of 5. Responses to the open-ended question included "My headaches have reduced dramatically...My quality of life is better...My mood improved...Personality has returned”, "I’ve completely changed as a person...I love life and it’s been fantastic", and "I wish this was around 30 years ago when I started getting headaches. My life would have been better”. A number of participants made comments to the effect that it was unfortunate that headache sufferers were not more commonly referred to psychologists.

\section{Conclusions}

The CBT program was associated with a significant reduction in headaches, and the decrease was significantly different from the RPC control group. The latter finding is unique as the only previous trial of an integrated treatment for comorbid headaches and depression failed to find a significant difference between headache changes in the treatment and control groups (Dindo et al., 2014). The reason for the different findings between the studies is not clear but the two studies did use quite different behavioral interventions as the intervention in Dindo et al. was a one-day Acceptance and Commitment Training plus Migraine Education workshop, as distinct from the 12 weekly 50-minute sessions of CBT based on a functional model used in this study.

The reduction in headaches from baseline to post-treatment of $42 \%$ is approximately in the middle of the range of 33\% to 55\% reported by Rains et al. (2005) for behavioral treatment of headache, but below the 68\% reported in our trial of CBT for recurrent headache 
(Martin et al., 2007). Presumably, the latter comparison reflects the negative impact of the comorbid major depressive disorder in this study.

The CBT program was associated with highly significant decreases on both measures of depression, and the decreases were significantly different from the control group. The effects for depression were larger than for headaches, consistent with the trial by Dindo and colleagues (Dindo et al., 2014; Dindo et al., 2012). The mean BDI-II score of the treatment group at baseline of 30.6 falls in the 'severe depression' category, and the mean score at posttreatment of 13.1 falls in the 'minimal to mild depression' category and below the cut-off for 'may need treatment' of 17 . These mean values therefore emphasize the clinical significance of the changes in depression.

The CBT program was associated with significant improvement, and differed significantly from the RPC group, on a measure of anxiety (even though not specifically targeted), and on multiple measures of quality of life, particularly the Mental Health subscale of the SF-36. Responses to the Feedback Questionnaire were generally very positive. There was no evidence of relapse in the four month follow-up data on any of the measures.

Six variables were explored as predictors of response to treatment, and only one emerged as statistically significant - men improved more than women. This is an interesting finding as reviews have reported that in trials of behavioral treatment of headache, women respond better than men (Holroyd \& Penzien, 1986). Reviews have reported that women do not respond less well to psychological treatment for depression than men (Parker \& Brotchie, 2010), so this does not explain the finding. Much evidence has accumulated demonstrating that major depressive disorder is far more common in females than males from around age 13 onwards (Essau, Lewinsohn, Seeley, \& Sasagawa, 2010), and researchers have developed theories to explain these gender differences. For example, one prominent cognitive theory of depression hypothesizes increased risk to and persistence of depression for individuals who 
ruminate about their depressed mood, in contrast to individuals who distract themselves from depressed mood (Nolen-Hoeksema, 1990), and there is evidence that females ruminate more than males (Hankin \& Abramson, 1999). This raises the possibility that ruminative response style may underlie the gender difference in treatment response found in this study.

Anxiety disorders were diagnosed in $71 \%$ of the sample, with an average of 2.0 anxiety disorders per participant. The literature on comorbid migraine and anxiety disorders has generally emphasized the association between migraine and panic disorder, generalized anxiety disorder, phobia and obsessive-compulsive disorder (Radat \& Swendsen, 2004; Baskin et al., 2006; Hamelsky \& Lipton, 2006), but studies have also reported associations between migraine and chronic daily headache on the one hand and posttraumatic stress disorder on the other (Peterlin et al., 2011). In this study, it was posttraumatic stress disorder that was diagnosed most often (36\% of the sample and 51\% of participants with anxiety disorders). This may reflect the fact that all participants in this study suffered from both headaches and major depressive disorder, and stressful life events can play a role in the development of both depression (Kendler, Karkowski, \& Prescott, 1999) and headaches (Henryk-Gutt \& Rees, 1973). Whilst it has been suggested that multiple psychiatric disorders predicts poorer response to headache treatment (Guidetti et al., 1998), there was no evidence in this trial that having an anxiety disorder as well as major depressive disorder resulted in less improvement in headaches.

Limitations of the study include the sample size. A larger sample would have been preferable but was not possible within the funds available. Nevertheless, significant differences were found on all the primary measures so that the small sample size merely testifies to the strength of these effects. It does mean that it is not possible to interpret nonsignificant findings as indicating that no effect existed as this could have resulted from inadequate statistical power. Thirty six participants were allocated to the intervention of 
whom seven declined to participate and eight withdrew from the intervention, resulting in 21 completing the intervention. This represents quite a high drop-out rate and should be taken into account in interpreting the findings. The drop-out rate probably reflects a number of factors including the length of the intervention at 12 sessions, and the fact that depression is associated with apathy, lethargy and indecisiveness, which may affect capacity to follow through a demanding intervention.

A longer follow-up period would have been desirable. Another limitation pertains to the fact that carrying out the intervention in the private practices of clinical psychologists working in the region made it more challenging to standardize treatment procedures, although we did provide training, Treatment Manuals, Client Handbooks, relaxation CDs, and required completion of check lists. The approach adopted did have the advantage that studies carried out in universities might not translate to clinical settings whereas this translational research study demonstrated that treatment could be administered through the Australian government healthcare system, with beneficial results to a recalcitrant patient group.

In terms of future research, it would be advantageous to conduct treatment trials on comorbid headaches and depression with larger samples. Longer term follow-up data than is currently available would be an advantage. Given the very high prevalence of anxiety disorders in individuals who suffer from headaches and depression, it seems logical to develop treatment packages that target all three disorders, although the counter argument is that presence of anxiety disorders did not predict poorer treatment outcome and the present treatment program resulted in significant improvement on a measure of anxiety severity. The finding that men with comorbid headaches and depression responded significantly better to behavioral treatment than women despite the reverse being true for behavioral treatment of headaches alone, seems worthy of further investigation. 
In summary, at baseline, the sample in this study were experiencing headaches at least 6 days per month, the average headache chronicity was 19 years, and $70 \%$ had more than one type of headache. All participants were diagnosed as major depressive disorder, and $71 \%$ also had at least one anxiety disorder (four participants had four or five anxiety disorders each). Despite these statistics emphasizing the multiple and severe disorders that participants in the sample experienced, comparing the CBT and RPC groups showed significant improvements in the treatment group relative to the control group in terms of headaches, depression, anxiety, and quality of life; and these changes were maintained at four month follow-up.

Acknowledgements: This research was supported by a grant from beyondblue Victorian Centre of Excellence, an initiative of beyondblue and the Victorian Government, administered through Monash University. Thanks to Crown House Publishing for giving permission to use a relaxation CD, as described in the text. Thanks to David Reutens, Leon Piterman, Martin Sharman, Lillian Nejad and Alex Wynd for contributions to the research, and to the clinical psychologists who provided treatment in this study. 


\section{References}

Altman, D. G. (2009). Missing outcomes in randomized trials: Addressing the dilemma. Open Medicine, 3, 51 - 53.

American Psychiatric Association. (2000). Practice guideline for the treatment of patients with major depressive disorder (revision). American Journal of Psychiatry, 157(Suppl), 1-45.

Andrasik, F., Lipchik, G. L., McCrory, D. C., \& Whitrock, D. A. (2005). Outcome measurement in behavioral research: Headache parameters and psychosocial outcome. Headache, 45, 429-437.

Baskin, S. M., \& Smitherman, T. A. (2011). Comorbidity between migraine and depression: update on traditional and alternative treatments. Neurological Sciences, 32 [Supp 1], S9-S13.

Beck, A. T., Epstein, N., Brown, G., \& Steer, R. A. (1988). An inventory for measuring clinical anxiety: Psychometric properties. Journal of Consulting and Clinical Psychology, 56, 893-897.

Beck, A. T., \& Steer, R. A. (1987). Manual for the revised BDI. San Antonio TX: The Psychological Corporation.

Bigal, M. E., \& Lipton, R. B. (2006). Modifiable risk factors for migraine progression. Headache, 46, 1334-1343.

Blanchard, E. B., Andrasik, F., Neff, D. F., Jurish, S., \& O’Keefe, D. M. (1981). Social validation of the headache diary. Behavior Therapy, 12, 711-715.

Breslau, N., Davis, G. C., \& Andreski, P. (1991). Migraine, psychiatric disorders, and suicide attempts: An epidemiologic study of young adults. Psychiatry Research, 37, 11-23.

Breslau, N., Davis, G. C., Schultz, L. R., \& Paterson, E. L. (1994). Migraine and Major Depression: A Longitudinal Study. Headache, 34, 387-393. 
Breslau, N., Lipton, R. B., Stewart, W. F., Schultz, L. R., \& Welch, K. M. A. (2003). Comorbidity of migraine and depression: Investigating potential etiology and prognosis. Neurology, 60, 1308-1312.

Buse, D. C., \& Andrasik, F. Behavioral medicine for migraine. (2009). Neurological Clinics, $27,445-465$.

Butler, A. C., Chapman, J. E., Forma, E. M., \& Beck, A. T. (2006). The empirical status of cognitive behavioral therapy. Clinical Psychology Review, 26, 17-31.

Collins, F. L., \& Thompson, J. K. (1979). Reliability and standardization in the assessment of self-reported head pain. Journal of Behavioral Assessment, 1, 73-86.

Dindo, L., Recober, A., Marchman, J., O’Hara, M. W., \& Turvey, C. (2014). One-day behavioral intervention in depressed migraine patients: Effects on headache. Headache, 54, 528-538.

Dindo, L., Recober, A., Marchman, J., Turvey, C., \& O’Hara, M. W. (2012). One-day behavioral treatment for patients with comorbid depression and migraine: A pilot study. Behaviour Research and Therapy, 50, 537-543.

Driesen, E., \& Hollon, S. D. (2010). Cognitive behavioral therapy for mood disorders: Efficacy, moderators and mediators. Psychiatry Clinics North America, 33, 537-555.

Essau, C. A., Lewinsohn, P. M., Seeley, J. R., \& Sasagawa, S. (2010). Gender differences in developmental course of depression. Journal of Affective Disorders, 127, 185-190.

Guidetti, V., Galli, F., Fabrizi, P., Giannantoni, A. S., Napoli, L., Bruni, O., \& Trillo, S. (1998). Headache and psychiatric comorbidity: clinical aspects and outcome in an 8year follow-up study. Cephalalgia, 18, 455-462.

Hamelsky, S. W., \& Lipton, R. B. (2006). Psychiatric comorbidity of migraine. Headache, $46,1327-1333$. 
Hankin, B. L., \& Abramson, L. Y. (1999). Development of gender differences in depression: Description and possible explanations. Annals Medicine, 31, 372-379.

Hawthorne, G., Richardson, J., \& Osborne, R. (1999). The Assessment of Quality of Life (AQoL) instrument: A psychometric measure of health-related quality of life. Quality Life Research, 8, 209-224.

Headache Classification Subcommittee of IHS. (2004). The International Classification of Headache Disorders, $2^{\text {nd }}$ ed. Cephalalgia, 24(Suppl 1), 1-151.

Henryk-Gutt, R., \& Rees, W. L. (1973). Psychological aspects of migraine. Journal of Psychosomatic Research, 17, 141-153.

Hollon, S. D., \& Ponniah, K. (2010). A review of empirically supported psychological therapies for mood disorders in adults. Depression Anxiety, 27, 891-932.

Holroyd, K. A., \& Penzien, D. B. (1986). Client variables and the behavioral treatment of recurrent tension headache: A meta-analytic review. Journal of Behavioral Medicine, 9, 515-536.

International Headache Society Committee on Clinical Trials in Tension-type Headache. (2000). Members’ Handbook. Oslo, Norway: Scandinavian University Press.

Juang, K-D., Wang, S-J., Fuh, J-L., Lu, S-R., \& Su, T-P. (2000). Comorbidity of depressive and anxiety disorders in chronic daily headache and its subtypes. Headache, 40, 818-823.

Kendler, K. S., Karkowski, L. M., \& Prescott, C. A. (1999). Causal relationship between stressful life events and the onset of major depression. American Journal of Psychiatry, 156, 837-841.

Kroenke, K., Spitzer, R. L., \& Williams, J. B. W. (2001). Validity of a brief depression severity measure. Journal of General Internal Medicine, 16, 606-613. 
Lake, A. E. (2006). Understanding the patient with complex psychiatric comorbidities: A psychologist’s perspective. Headache, 46(Suppl 3), S157-S167.

Lake, A. E., Rains, J. C., Penzien, D. B., \& Lipchik, G. L. (2005). Headache and psychiatric comorbidity: Historical context, clinical implications, and research relevance. Headache, 45, 493-506.

Lean, M. E. J., Mann, J. I., Hoek, J. A., Elliot, R. M., \& Schofield, G. (2008). Translational research: From evidence based medicine to sustainable solutions for public health problems. British Medical Journal, 337, 705-706.

Lewinsohn, P. M., Antonuccio, D. O, Steinmetz, J. L., \& Teri, L. (1984). The coping with depression course: A psychoeducational intervention for unipolar depression. Eugene, OR: Castala.

Lipchik, G. L., \& Penzien, D. B. (2004). Psychiatric comorbidities in patients with headaches. Seminar Pain Medicine, 2, 93-105.

Lipchik, G. L., Smitherman, T. A., Penzien, D. B., \& Holroyd, K. A. (2006). Basic principles of cognitive-behavioral therapies for comorbid psychiatric symptoms among headache patients. Headache, 46(Suppl 3), S119-S132.

Marazziti, D., Toni, C., Pedri, S., Bonuccelli, U., Pavese, N., Nuti, A.,...Akiskal, H. S. (1995). Headache, panic disorder and depression: Comorbidity or a spectrum? Neuropsychobiology, 31, 125-129.

Martin, P. R. (1993). Psychological management of chronic headaches. New York: Guilford Press.

Martin, P. R. (2013). Psychological management of the common primary headaches. In M. L. Caltabiano \& L. A. Ricciardelli (Eds.). Applied topics in health psychology (pp. 462476). Wiley-Blackwell. 
Martin, P. R., Forsyth, M. R., \& Reece, J. (2007). Cognitive-behavioral therapy versus temporal pulse amplitude biofeedback training for recurrent headache. Behavior Therapy, 38, 350-363.

Milgrom, J., Martin, P. R., \& Negri, L. (1999). Treating postnatal depression: A psychological approach for health care practitioners. Chichester: John Wiley.

Mongini, F., Keller, R., Deregibus, A., Raviola, F., Mongini, T., \& Sancarlo, M. (2003). Personality traits, depression and migraine in women: A longitudinal study. Cephalalgia, 23, 186-192.

Nejad, L., \& Volny, K. (2008). Treating stress and anxiety: A practitioner's guide to evidence-based approaches. Carmarthen, Wales: Crown House Publishing.

Nolen-Hoeksema, S. (1990). Sex differences in depression. Stanford, CA: Stanford University Press.

Norman, G. (2010). Likert scales, levels of measurement, and the 'laws' of statistics. Advances in Health Sciences Education, 15, 625-632.

Parker, G., \& Brotchie, H. (2010). Gender differences in depression. International Review of Psychiatry, 22, 429-436.

Penzien, D. B., Andrasik, F., Freidenberg, B. M., Houle, T. T., Lake, A. E., Lipchik, G. L.,...Wittrock, D. A. (2005). Guidelines for trials of behavioral treatments for recurrent headache, first edition: American Headache Society Behavioral Clinical Trials Workgroup. Headache, 45(Suppl 2), S110-S132.

Peterlin, B. L., Rosso, A. L., Sheftell, F. D., Libon, D. J., Mossey, J. M., \& Merikangas, K. R. (2011). Post-traumatic stress disorder, drug abuse and migraine. New findings from the National Comorbidity Survey Replication (NCS-R). Cephalalgia, 31, 235-244.

Pompili, M., Di Cosimo, D., Innamorati, M., Lester, D., Tatarelli, R., \& Martelletti, P. (2009). Psychiatric comorbidity in patients with chronic daily headache and 
migraine: a selective overview including personality traits and suicide risk. Journal of Headache Pain, 10, 283-290.

Radat, F., Creac'h, C., Swendsen, J. D., Lafittau, M., Irachabal, S., Dousset, V., \& Henry, P. (2005). Psychiatric comorbidity in the evolution from migraine to medication overuse headache. Cephalalgia, 25, 519-522.

Radat, F., \& Swendsen, J. (2004). Psychiatric comorbidity in migraine: A review. Cephalalgia, 25, 165-178.

Rains, J. C., Penzien, D. B., McCrory, D. C., \& Gray, R. N. (2005). Behavioral headache treatment: History, review of the empirical literature, and methodological critique. Headache, 45(Suppl 2), S92-S109.

Schur, E. A., Noonan, C., Buchwald, D., Goldberg, J., \& Afari, N. (2009). A Twin Study of Depression and Migraine: Evidence for a Shared Genetic Vulnerability. Headache, 49, 1493-1502.

Smitherman, T. A., McDermott, M. J., \& Buchanan, E. M. (2011). Negative impact of episodic migraine on a university population: Quality of life, functional impairment, and comorbid psychiatric symptoms. Headache, 51, 581-589.

Ware, J.E., Jr., Gandek, B., IQOLA Project Group. (1994). The SF-36 Health Survey: Development and Use in Mental Health Research and IQOLA Project. International Journal of Mental Health, 23, 49-73.

World Health Organization Collaborating Centre for Mental Health and Substance Abuse. (1997). Composite International Diagnostic Interview: CIDI-Auto, Version 2.1 (computer program). WHO, Geneva.

Wynd, A., Martin, P. R., Gilson, K., \& Meadows, G. N. (in press). Investigating the relationship between comorbid headaches and depression. Australian Psychologist. 
Completed telephone screen to assess initial eligibility $(n=399)$

Excluded from the study $(n=300)$

- Not meeting inclusion criteria $(n=253)$

- Eligible but declined participation $(n=38)$

- Changed situation; no longer meeting criteria before first assessment $(n=9)$

completed first

assessment $(n=99)$

Excluded from the study $(n=24)$

- Not meeting inclusion criteria $(n=13)$

- Declined to participate $(n=11)$

Completed second

assessment $(n=75)$

Excluded from the study $(n=9)$

- Not meeting inclusion criteria $(n=9)$

- Declined to participate $(n=0)$

Randomized $(n=66)$

Allocated to CBT intervention $(n=36)$

- Received CBT intervention $(n=21)$

- Declined CBT intervention ( $n=7)$. Reasons: moved interstate, new job, personal reasons, too busy, unable to commit to study.

- Withdrew during CBT intervention $(n=8)$.

Completed Post Assessment $(n=21)$

- Discontinued intervention ( $n=8)$. Reasons: inpatient admission, pregnancy, feeling better, child unwell, moved away, unknown.

- Did CBT but did not complete follow-up $(n=0)$

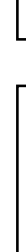

Completed 4 month Follow-Up $(n=19)$

- Lost to follow-up ( $n=1)$

- Study completed before follow-up $(n=1)$

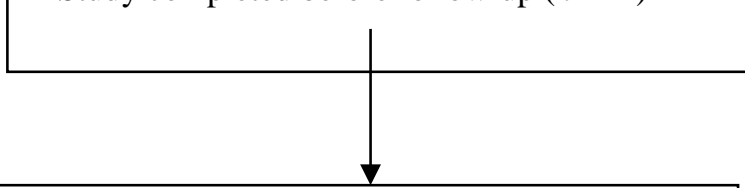

Analyzed $(n=36)$

- No participants excluded from analysis
Allocated to RPC control $(n=30)$

- Completed RPC control $(n=25)$

- Did not complete RPC control $(n=5)$.

Reasons: did not accept allocation $(n=1)$, not contactable after RPC ( $n=1)$, withdrew during RPC $(n=2)$, lost diaries $(n=1)$.

Completed Post Assessment $(n=25)$

- Did not complete allocation $(n=5)$

- Did not complete follow-up $(n=0)$

Analyzed $(n=30)$

- No participants excluded from analysis 


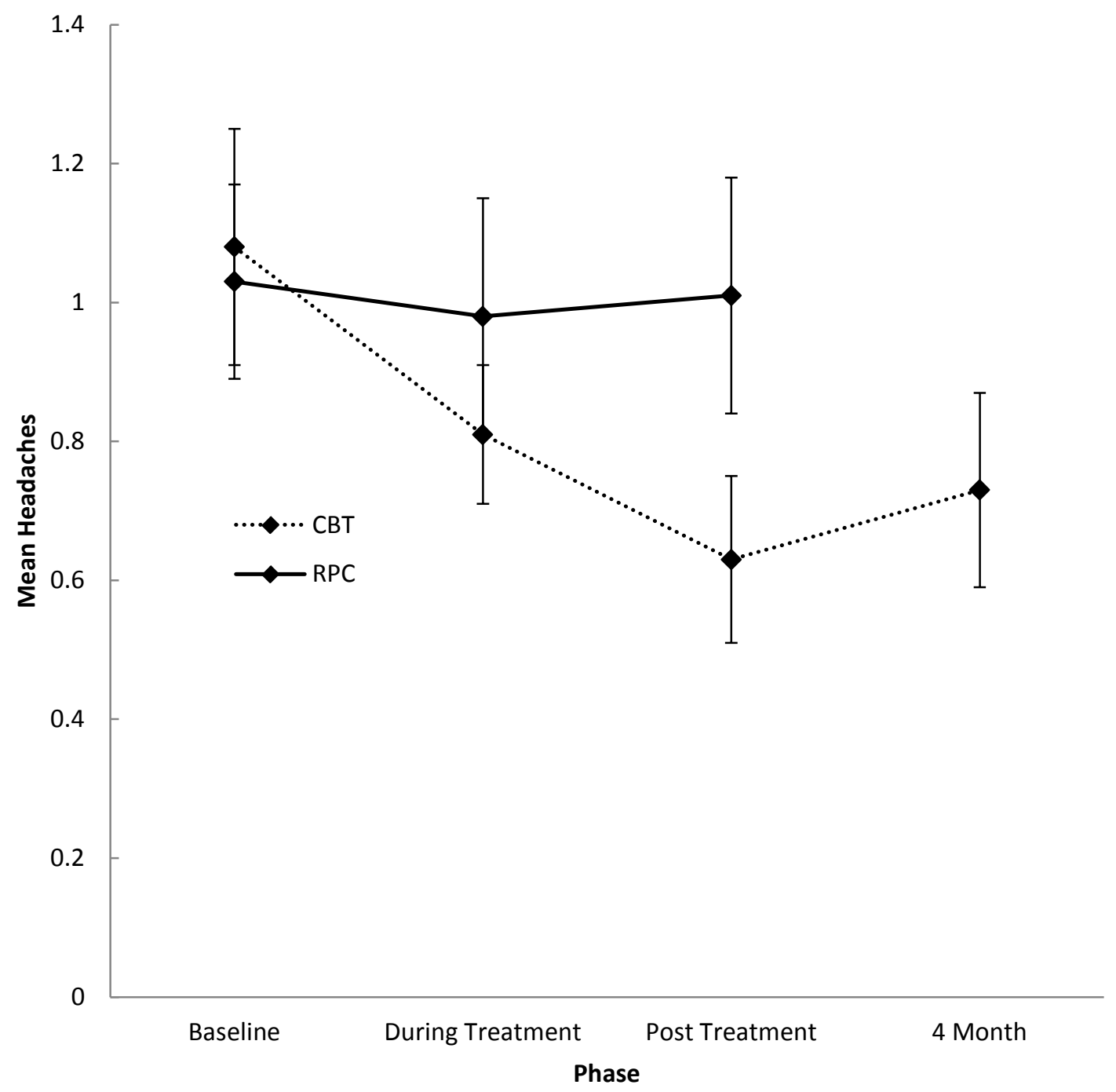

Figure 2. Mean daily headache ratings as a function of treatment and phase. Error bars shows 95\% confidence intervals. 

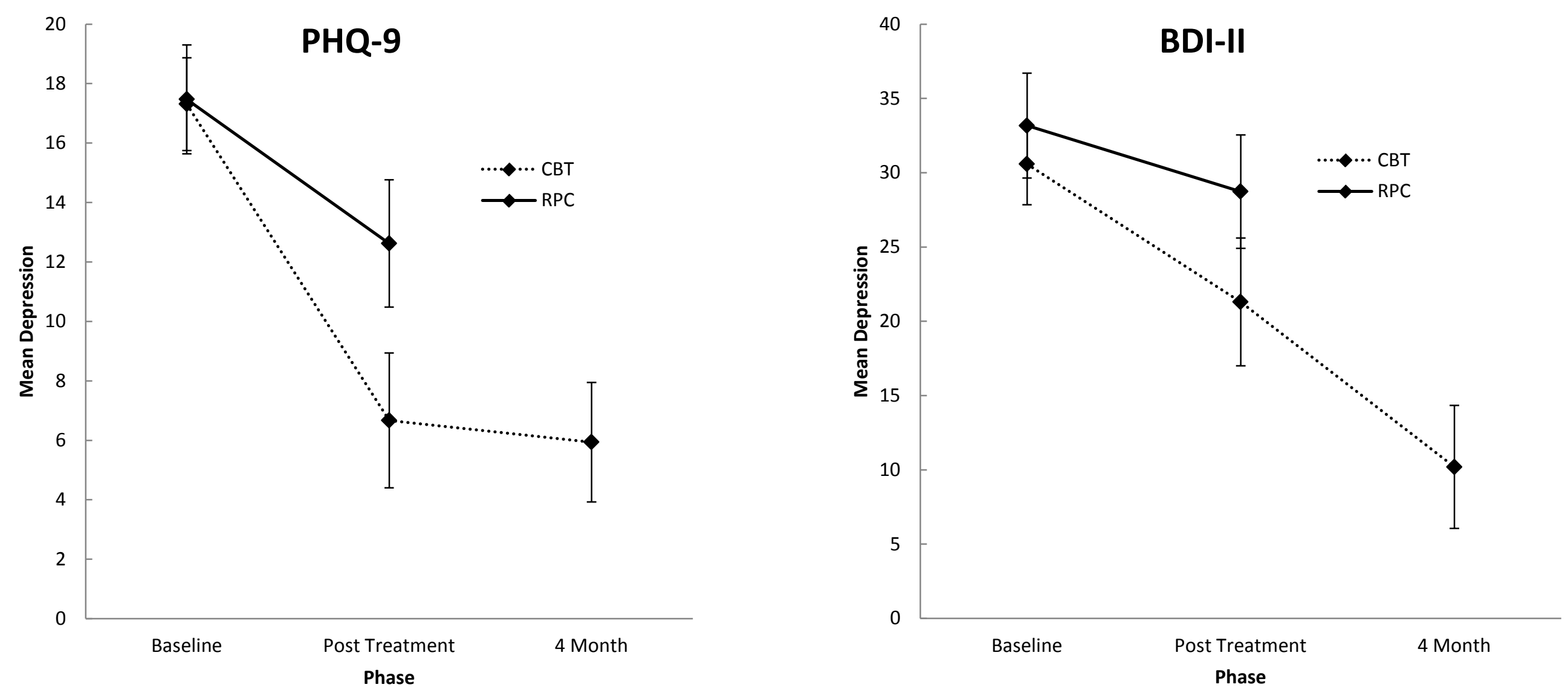

Figure 3. Mean depression scores as a function of treatment and phase for two measures of depression. Error bars shows $95 \%$ confidence intervals. 
Table 1

Demographic and Clinical Characteristics of Sample.

\begin{tabular}{lllll}
\hline Variable & & Group & \\
\cline { 3 - 3 } & RPC & CBT & Total \\
\hline
\end{tabular}

Gender

Male

$\begin{array}{lll}8 & 9 & 17\end{array}$

Female

22

27

17

Age in years

$\begin{array}{llrr}M & 40.83 & 40.19 & 40.64 \\ S D & 14.32 & 12.89 & 13.41\end{array}$

Education

Less than Year 12 (senior year in N America)

Year 12

$\begin{array}{ccc}1 & 5 & 6 \\ 6 & 5 & 11 \\ 9 & 7 & 16 \\ 8 & 12 & 20 \\ 6 & 7 & 13\end{array}$

Occupation

Certificate/Diploma

Undergraduate degree

Managers

Professionals

Community \& Personal Service Workers

Other employment categories ${ }^{1}$

Not employed

$\begin{array}{ccc}5 & 0 & 5 \\ 7 & 11 & 18 \\ 4 & 3 & 7 \\ 2 & 5 & 7 \\ 5 & 10 & 15 \\ 3 & 2 & 5 \\ 4 & 5 & 9\end{array}$

Retired

Student

Headache diagnosis

Migraine with Aura

$\begin{array}{ccc}6 & 9 & 15 \\ 11 & 10 & 21 \\ 2 & 6 & 8 \\ 1 & 1 & 2 \\ 10 & 10 & 20\end{array}$

Headache chronicity in years

$M$

1،Other employment categories’ includes Clerical \& Administrative Workers, Technicians \& Trades Workers, Sales Workers, Machinery Operators \& Drivers, and Labourers.

\footnotetext{
2 Types of anxiety disorders present were (number of cases in parentheses): Posttraumatic Stress Disorder (24), Specific Phobia (22), Panic Disorder Without Agoraphobia (15), Panic Disorder With Agoraphobia (14), Social Phobia (14), Generalized Anxiety Disorder (3), Obsessive-Compulsive Disorder (3), and Agoraphobia Without History of Panic Disorder (1).
} 
Table 2

Descriptive Statistics and Analysis of Variance Results for Experimental Groups on Outcome Measures.

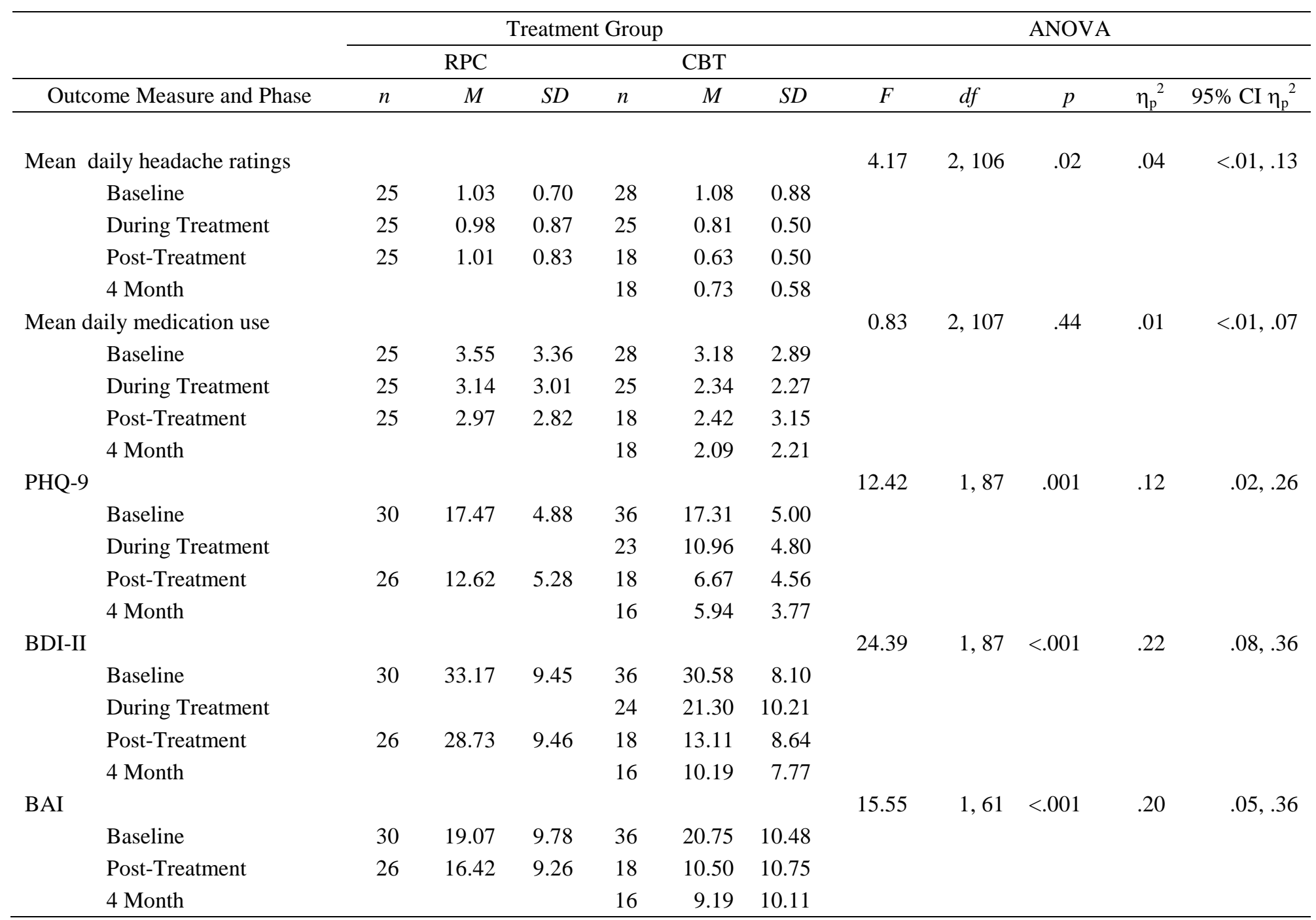


Table 2 (continued)

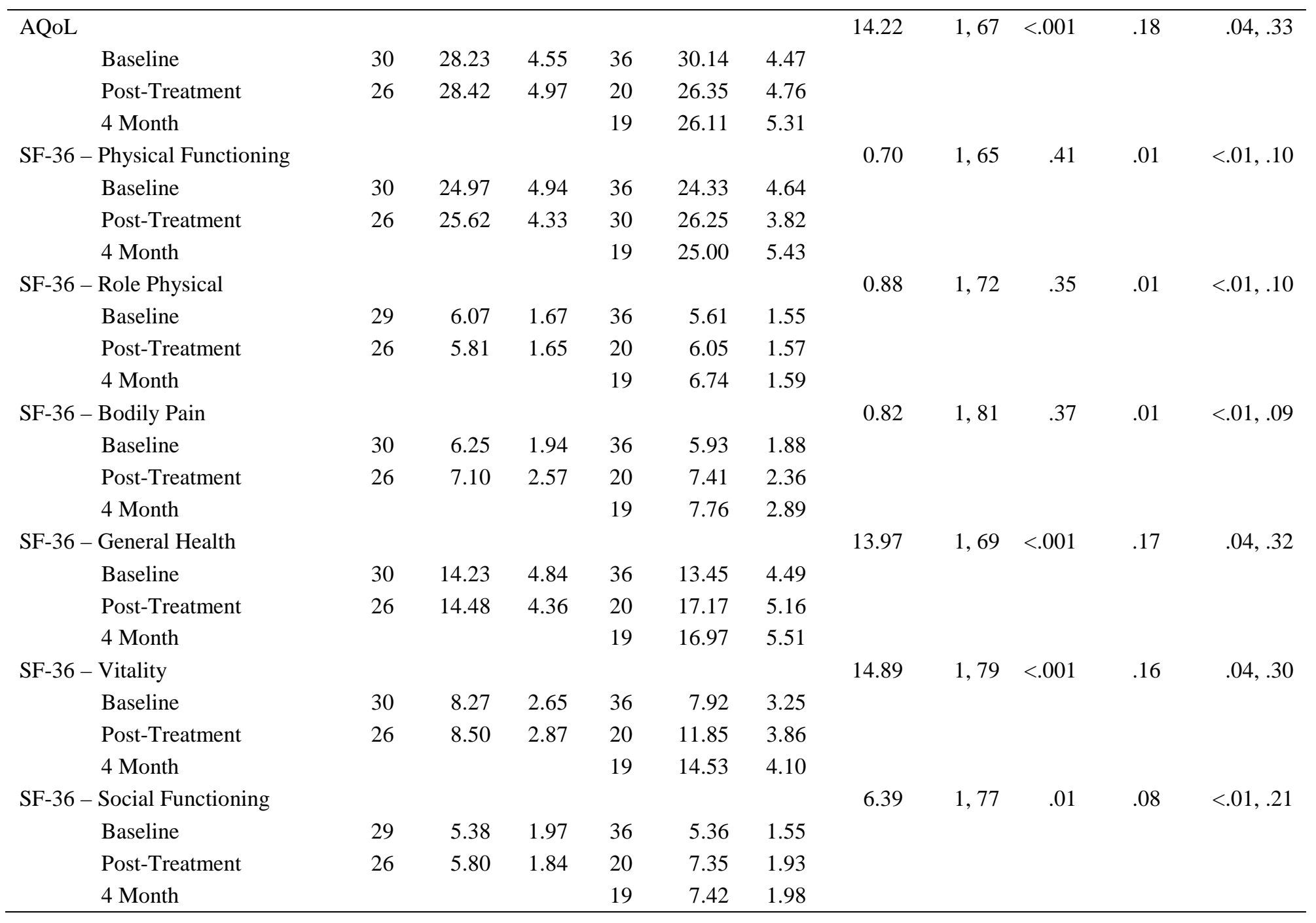


Table 2 (continued)

\begin{tabular}{|c|c|c|c|c|c|c|c|c|c|c|c|}
\hline SF-36 - Role Emotional & & & & & & & 6.42 & 1,82 & .01 & .07 & $<.01, .20$ \\
\hline Baseline & 29 & 3.44 & 0.83 & 36 & 3.64 & 0.80 & & & & & \\
\hline Post-Treatment & 26 & 3.65 & 0.89 & 20 & 4.80 & 1.24 & & & & & \\
\hline SF-36 - Mental Health & & & & & & & 15.62 & 1,78 & $<.001$ & .17 & $.04, .31$ \\
\hline Baseline & 30 & 13.10 & 3.10 & 36 & 12.97 & 3.62 & & & & & \\
\hline Post-Treatment & 26 & 14.88 & 4.17 & 20 & 19.10 & 3.97 & & & & & \\
\hline
\end{tabular}

Note. ANOVA's are the Group by Phase interaction result for each measure. Fractional denominator degrees of freedom have been rounded to the nearest whole. 
Table 3

Simple Main Effects Analysis for Treatment Within Phase for Significant Interaction Results.

\begin{tabular}{lrrrrrrr}
\hline & \multicolumn{7}{c}{ Phase } \\
\multicolumn{1}{c}{ Baseline } & \multicolumn{2}{c}{ Post-Treatment } \\
\hline \multicolumn{1}{c}{ Outcome Measure } & \multicolumn{1}{c}{$p$} & \multicolumn{1}{c}{$d$} & $95 \%$ CI $d$ & $p$ & $d$ & $95 \%$ CI $d$ \\
\hline Mean daily headache ratings & .81 & 0.07 & $0.03,0.10$ & .04 & 0.66 & $0.33,0.99$ \\
PHQ-9 & .89 & 0.03 & $0.02,0.05$ & $<.001$ & 1.16 & $0.58,1.74$ \\
BDI-II & .25 & 0.28 & $0.14,0.43$ & $<.001$ & 1.82 & $0.91,2.73$ \\
BAI & .50 & 0.17 & $0.08,0.25$ & .03 & 0.68 & $0.34,1.02$ \\
AQoL & .11 & 0.41 & $0.20,0.61$ & .18 & 0.40 & $0.20,0.61$ \\
SF-36 - General Health & .27 & 0.28 & $0.14,0.41$ & .08 & 0.52 & $0.26,0.78$ \\
SF-36 - Vitality & .66 & 0.11 & $0.05,0.16$ & $<.001$ & 1.07 & $0.54,1.61$ \\
SF-36 - Social Functioning & .99 & $<0.01$ & $<0.01,0.01$ & .008 & 0.80 & $0.40,1.20$ \\
SF-36 - Role Emotional & .44 & 0.21 & $0.10,0.31$ & $<.001$ & 1.07 & $0.54,1.61$ \\
SF-36 - Mental Health & .89 & 0.03 & $0.02,0.05$ & $<.001$ & 1.21 & $0.61,1.82$ \\
\hline
\end{tabular}

